\title{
RINGS OF INVARIANT MODULE TYPE AND AUTOMORPHISM-INVARIANT MODULES
}

\author{
SURJEET SINGH AND ASHISH K. SRIVASTAVA
}

Dedicated to T. Y. Lam on his 70 th Birthday

\begin{abstract}
A module is called automorphism-invariant if it is invariant under any automorphism of its injective hull. In [Algebras for which every indecomposable right module is invariant in its injective envelope, Pacific J. Math., vol. 31, no. 3 (1969), 655-658] Dickson and Fuller had shown that if $R$ is a finite-dimensional algebra over a field $\mathbb{F}$ with more than two elements then an indecomposable automorphism-invariant right $R$-module must be quasiinjective. In this paper we show that this result fails to hold if $\mathbb{F}$ is a field with two elements. Dickson and Fuller had further shown that if $R$ is a finitedimensional algebra over a field $\mathbb{F}$ with more than two elements, then $R$ is of right invariant module type if and only if every indecomposable right $R$-module is automorphism-invariant. We extend the result of Dickson and Fuller to any right artinian ring. A ring $R$ is said to be of right automorphism-invariant type (in short, RAI-type) if every finitely generated indecomposable right $R$ module is automorphism-invariant. In this paper we completely characterize an indecomposable right artinian ring of RAI-type.
\end{abstract}

\section{INTRODUCTION}

All our rings have identity element and modules are right unital. A right $R$-module $M$ is called an automorphism-invariant module if $M$ is invariant under any automorphism of its injective hull, i.e. for any automorphism $\sigma$ of $E(M), \sigma(M) \subseteq M$ where $E(M)$ denotes the injective hull of $M$.

Indecomposable modules $M$ with the property that $M$ is invariant under any automorphism of its injective hull were first studied by Dickson and Fuller in [5] for the particular case of finite-dimensional algebras over fields $\mathbb{F}$ with more than two elements. But for modules over arbitrary rings, study of such a property has been initiated recently by Lee and Zhou in 14. The dual notion of these modules has been proposed by Singh and Srivastava in [17.

The obvious examples of the class of automorphism-invariant modules are quasiinjective modules and pseudo-injective modules. Recall that a module $M$ is said to be $N$-injective if for every submodule $N_{1}$ of the module $N$, all homomorphisms $N_{1} \rightarrow M$ can be extended to homomorphisms $N \rightarrow M$. A right $R$-module $M$ is injective if $M$ is $N$-injective for every $N \in \operatorname{Mod}-R$. A module $M$ is said to be

2000 Mathematics Subject Classification. 16U60, 16D50.

Key words and phrases. rings of invariant module type, automorphism-invariant modules, quasi-injective modules, pseudo-injective modules. 
quasi-injective if $M$ is $M$-injective. A module $M$ is called pseudo-injective if every monomorphism from a submodule of $M$ to $M$ extends to an endomorphism of $M$.

Thus we have the following hierarchy;

injective $\Longrightarrow$ quasi-injective $\Longrightarrow$ pseudo-injective $\Longrightarrow$ automorphism-invariant

It is well known that a quasi-injective module need not be injective. In 18 Teply gave construction of a pseudo-injective module which is not quasi-injective. We do not know yet an example of an automorphism-invariant module which is not pseudo-injective.

Dickson and Fuller [5] studied automorphism-invariant modules in case of finitedimensional algebras over a field $\mathbb{F}$ with more than two elements. They proved that if $R$ is a finite-dimensional algebra over a field $\mathbb{F}$ with more than two elements then an indecomposable automorphism-invariant right $R$-module must be quasiinjective. We show that this result fails to hold if $\mathbb{F}$ is a field with two elements. A ring $R$ is said to be of right invariant module type if every indecomposable right $R$-module is quasi-injective. Dickson and Fuller had further shown that if $R$ is a finite-dimensional algebra over a field $\mathbb{F}$ with more than two elements, then $R$ is of right invariant module type if and only if every indecomposable right $R$-module is automorphism-invariant. We extend the result of Dickson and Fuller to any right artinian ring.

We call a ring $R$ to be of right automorphism-invariant type (in short, RAItype), if every finitely generated indecomposable right $R$-module is automorphisminvariant. In this paper we study the structure of indecomposable right artinian rings of RAI-type.

Lee and Zhou in 14 asked whether every automorphism-invariant module is pseudo-injective. In this paper we show that the answer is in the affirmative for modules with finite Goldie dimension.

We also prove that a simple right noetherian ring $R$ is a right SI ring if and only if every cyclic singular right $R$-module is automorphism-invariant.

Before presenting the proofs of these results, let us recall some basic definitions and facts. A module $M$ is said to have finite Goldie (or uniform) dimension if it does not contain an infinite direct sum $\bigoplus_{n \in \mathbb{N}} M_{n}$ of non-zero submodules.

A module $M$ is called directly-finite if $M$ is not isomorphic to a proper summand of itself. Clearly, a module with finite Goldie dimension is directly-finite. A module $M$ is called a square if $M \cong X \oplus X$ for some module $X$; and a module is called square-free if it does not contain a non-zero square.

A module $M$ is said to have the internal cancellation property if whenever $M=$ $A_{1} \oplus B_{1}=A_{2} \oplus B_{2}$ with $A_{1} \cong A_{2}$, then $B_{1} \cong B_{2}$. For details on internal cancellation property, the reader is referred to [13. Now, if an injective module $M$ is directlyfinite, then it has internal cancellation property (see [15, Theorem 1.29]).

A module $M$ is said to be uniserial if any two submodules of $M$ are comparable with respect to inclusion. A ring $R$ is called a right uniserial ring if $R_{R}$ is a uniserial module. Any direct sum of uniserial modules is called a serial module. A ring $R$ is said to be a right serial ring if the module $R_{R}$ is serial. A ring $R$ is called a serial ring if $R$ is both left as well as right serial. 
If $A$ is an essential submodule of $B$, then we denote it as $A \subseteq_{e} B$. For any module $M$, we define $Z(M)=\left\{x \in M: \operatorname{ann}_{r}(x) \subseteq_{e} R_{R}\right\}$. It can be easily checked that $Z(M)$ is a submodule of $M$. It is called the singular submodule of $M$. If $Z(M)=M$, then $M$ is called a singular module. If $Z(M)=0$, then $M$ is called a non-singular module.

Consider the following three conditions on a module $M$;

C1: Every submodule of $M$ is essential in a direct summand of $M$.

C2: Every submodule of $M$ isomorphic to a direct summand of $M$ is itself a direct summand of $M$.

C3: If $N_{1}$ and $N_{2}$ are direct summands of $M$ with $N_{1} \cap N_{2}=0$ then $N_{1} \oplus N_{2}$ is also a direct summand of $M$.

A module $M$ is called a continuous module if it satisfies conditions $\mathrm{C} 1$ and $\mathrm{C} 2$. A module $M$ is called $\pi$-injective (or quasi-continuous) if it satisfies conditions $\mathrm{C} 1$ and C3. A module $M$ is called a CS module (or extending module) if it satisfies condition $\mathrm{C} 1$.

In general, we have the following implications.

$$
\text { Injective } \Longrightarrow \text { Quasi-injective } \Longrightarrow \text { Continuous } \Longrightarrow \pi \text {-injective } \Longrightarrow \mathrm{CS}
$$

The socle of a module $M$ is denoted by $\operatorname{Soc}(M)$. A right $R$-module $M$ is called semi-artinian if for every submodule $N \neq M$, $\operatorname{Soc}(M / N) \neq 0$. A ring $R$ is called right semi-artinian if $R_{R}$ is semi-artinian. We denote by $J(R)$, the Jacobson radical of a ring $R$. For any term not defined here, the reader is referred to [9], 11], 12, and [15].

\section{Basic Facts about Automorphism-invariant Modules}

Lee and Zhou proved the following basic facts about automorphism-invariant modules [14.

- A module $M$ is automorphism-invariant if and only if every isomorphism between any two essential submodules of $M$ extends to an automorphism of $M$.

- A direct summand of an automorphism-invariant module is automorphisminvariant.

- If for two modules $M_{1}$ and $M_{2}, M_{1} \oplus M_{2}$ is automorphism-invariant, then $M_{1}$ is $M_{2}$-injective and $M_{2}$ is $M_{1}$-injective.

- Every automorphism-invariant module satisfies the property C3.

- A CS automorphism-invariant module is quasi-injective. 


\section{RESULTS}

Dickson and Fuller in [5] considered a finite-dimensional algebra $R$ over a field $\mathbb{F}$ with more than two elements and proved that if an indecomposable right $R$-module $M$ is automorphism-invariant, then $M$ is quasi-injective. They further obtained the following.

Theorem 1. (Dickson and Fuller, [5]) Let $R$ be a finite-dimensional algebra over a field $\mathbb{F}$ with more than two elements. Then the following statements are equivalent;

(i) Each indecomposable right $R$-module is automorphism-invariant.

(ii) Each indecomposable right $R$-module is quasi-injective.

(iii) Each indecomposable right $R$-module has a square-free socle.

We will provide an example to show that if $R$ is a finite-dimensional algebra over a field $\mathbb{F}$ with two elements, then an indecomposable automorphism-invariant right $R$-module need not be quasi-injective.

First, note that in an artinian serial ring $R$, any indecomposable summand of $R_{R}$ of maximum length is injective. Thus if $T_{n}(D)$ is the upper triangular matrix ring over a division ring $D$, then $e_{11} T_{n}(D)$ is injective and uniserial.

Example. Let $R=\left[\begin{array}{ccc}\mathbb{F} & \mathbb{F} & \mathbb{F} \\ 0 & \mathbb{F} & 0 \\ 0 & 0 & \mathbb{F}\end{array}\right]$ where $\mathbb{F}$ is a field of order 2 .

We know that $R$ is a left serial ring. Note that $e_{11} R$ is a local module, $e_{12} \mathbb{F} \cong e_{22} R$, $e_{13} \mathbb{F} \cong e_{33} R$ and $e_{11} J(R)=e_{12} \mathbb{F} \oplus e_{13} \mathbb{F}$, a direct sum of two minimal right ideals. So the injective hull of $e_{11} R$ is $E\left(e_{11} R\right)=E_{1} \oplus E_{2}$, where $E_{1}=E\left(e_{12} \mathbb{F}\right)$ and $E_{2}=E\left(e_{13} \mathbb{F}\right)$.

Now set $A=\operatorname{ann}_{r}\left(e_{12} \mathbb{F}\right)$. Then $A=e_{13} \mathbb{F}+e_{33} \mathbb{F}$. Thus $\bar{R}=R / A \cong\left[\begin{array}{cc}\mathbb{F} & \mathbb{F} \\ 0 & \mathbb{F}\end{array}\right]=$ $S$. Denote the first row of $S$ by $S_{1}$. It may be checked that $S_{1}$ is injective. As $\mathbb{F}$ has only two elements, $S_{1}$ has only two endomorphisms, zero and the identity. Take the pre-image $L_{1}$ of $S_{1}$ in $\bar{R}$. It is uniserial with composition length 2 , and $e_{12} \mathbb{F}$ naturally embeds in $L_{1}$. There is no mapping of $e_{13} \mathbb{F}$ into $L_{1}$. It follows that $L_{1}$ is $e_{11} R$-injective and $e_{12} \mathbb{F}$-injective. As $e_{22} R \cong e_{12} \mathbb{F}, L_{1}$ is $e_{22} R$-injective. There is no map from $e_{33} R$ into $L_{1}$ so it is also $e_{33} R$-injective. Hence $L_{1}$ is injective. Thus $E_{1}=L_{1}$ and its ring of endomorphisms has only two elements.

If $B=\operatorname{ann}_{r}\left(e_{13} \mathbb{F}\right)$, then $B=e_{12} \mathbb{F}+e_{22} \mathbb{F}$. Thus $R / B \cong\left[\begin{array}{cc}\mathbb{F} & \mathbb{F} \\ 0 & \mathbb{F}\end{array}\right]$. The preimage of $S_{1}$ in $R / B$ is $L_{2}$, which is uniserial, and injective. We have $E_{2} \cong L_{2}$ and its ring of endomorphism has only two elements.

Note that $e_{11} R$ has all its composition factors non-isomorphic, both $L_{1}$ and $L_{2}$ have composition length 2 with $\frac{L_{1}}{L_{1} J(R)} \cong \frac{e_{11} R}{e_{11} J(R)}, L_{1} J(R) \cong e_{22} R, \frac{L_{2}}{L_{2} J(R)} \cong$ $\frac{e_{11} R}{e_{11} J(R)}$, and $L_{2} J(R) \cong e_{33} R$. Thus $L_{1}, L_{2}$ have isomorphic tops but non-isomorphic socles.

Suppose there exists a non-zero mapping $\sigma: L_{1} \rightarrow L_{2}$. Then $\sigma\left(L_{1}\right)=L_{2} J(R)$. Thus $\frac{e_{11} R}{e_{11} J(R)} \cong e_{33} R$, which is a contradiction. Therefore, there is no non-zero map between $L_{1}$ and $L_{2}$. 
Hence the only automorphism of $L_{1} \oplus L_{2}$ is the identity. So $e_{11} R$ is trivially automorphism-invariant but it is not uniform. Then clearly $e_{11} R$ is not quasiinjective as an indecomposable quasi-injective module must be uniform.

Thus, this ring $R$ is an example of a finite-dimensional algebra over a field $\mathbb{F}$ with two elements such that there exists an indecomposable right $R$-module which is automorphism-invariant but not quasi-injective.

Next, we proceed to extend the result of Dickson and Fuller [5] to any right artinian ring. But, first we obtain a useful result on decomposition property of automorphism-invariant modules.

We will show that under certain conditions a decomposition of injective hull $E(M)$ of an automorphism-invariant module $M$ induces a natural decomposition of $M$.

We will denote the identity automorphism on any module $M$ by $I_{M}$.

Lemma 2. Let $M$ be an automorphism-invariant right module over any ring $R$. If $E(M)=E_{1} \oplus E_{2}$ and $\pi_{1}: E(M) \rightarrow E_{1}$ is an associated projection, then $M_{1}=$ $\pi_{1}(M)$ is also automorphism-invariant.

Proof. Let $E(M)=E_{1} \oplus E_{2}$ and $M_{1}=\pi_{1}(M)$, where $\pi_{1}: E(M) \rightarrow E_{1}$ is a projection with $E_{2}$ as its kernel. Let $\sigma_{1}$ be an automorphism of $E_{1}$ and $x_{1} \in M_{1}$. For some $x \in M$, and $x_{2} \in E_{2}$, we have $x=x_{1}+x_{2}$. Now $\sigma=\sigma_{1} \oplus I_{E_{2}}$ is an automorphism of $E$. Thus $\sigma(x)=\sigma_{1}\left(x_{1}\right)+x_{2} \in M$, which gives $\sigma_{1}\left(x_{1}\right) \in M_{1}$. Hence $M_{1}$ is automorphism-invariant.

Lemma 3. Let $M$ be an automorphism-invariant right module over any ring $R$. Let $E(M)=E_{1} \oplus E_{2}$ such that there exists an automorphism $\sigma_{1}$ of $E_{1}$ such that $I_{E_{1}}-\sigma_{1}$ is also an automorphism of $E_{1}$. Then

$$
M=\left(M \cap E_{1}\right) \oplus\left(M \cap E_{2}\right) .
$$

Proof. Set $E=E(M)$. Set $I_{E}=I_{E_{1}} \oplus I_{E_{2}}$, and $\sigma=\sigma_{1} \oplus I_{E_{2}}$. Clearly, both $I_{E}$ and $\sigma$ are automorphisms of $E$. Since $M$ is assumed to be an automorphisminvariant module, $M$ is invariant under automorphisms $I_{E}$ and $\sigma$. Consequently, $M$ is invariant under $I_{E}-\sigma$ too. Note that $I_{E}-\sigma=\left(I_{E_{1}}-\sigma_{1}\right) \oplus 0$. Thus $\left(I_{E}-\sigma\right)(M)=\left(I_{E_{1}}-\sigma_{1}\right)(M) \subseteq M$. Let $\pi_{1}: E \rightarrow E_{1}$ and $\pi_{2}: E \rightarrow E_{2}$ be the canonical projections. Set $M_{1}=\pi_{1}(M)$ and $M_{2}=\pi_{2}(M)$. Now $M \cap E_{1} \subseteq M_{1}$ and $M \cap E_{2} \subseteq M_{2}$.

Let $0 \neq u_{1} \in E_{1}$. For some $r \in R, 0 \neq u_{1} r \in M$ and thus $u_{1} r \in M_{1}$. Thus $M_{1} \subseteq_{e} E_{1}$. By Lemma 2, $M_{1}$ is automorphism-invariant. Therefore, $M_{1}=\left(I_{E_{1}}-\right.$ $\left.\sigma_{1}\right)^{-1}\left(M_{1}\right)$. Let $x_{1} \in M_{1}$. Then, we have for some $x \in M, x=x_{1}+x_{2}, x_{2} \in E_{2}$. Now, as $I_{E_{1}}-\sigma_{1}$ is an automorphism on $E_{1}$, there exists an element $y_{1} \in E_{1}$ such that $\left(I_{E_{1}}-\sigma_{1}\right)\left(y_{1}\right)=x_{1}$, which gives $y_{1} \in\left(I_{E_{1}}-\sigma_{1}\right)^{-1}\left(M_{1}\right)=M_{1}$. This yields an element $y \in M$ such that $y=y_{1}+y_{2}$ for some $y_{2} \in E_{2}$. We get $\left(I_{E}-\sigma\right)(y)=\left(I_{E_{1}}-\sigma_{1}\right)\left(y_{1}\right)=x_{1}$. Thus $x_{1} \in\left(I_{E}-\sigma\right)(M)$. As $\left(I_{E}-\sigma\right)(M) \subseteq M$, we get $x_{1} \in M$. Hence $M_{1} \subseteq M$.

Now, let $u_{2} \in M_{2}$ be an arbitrary element. For some $u_{1} \in M_{1}$, we have $u=$ $u_{1}+u_{2} \in M$. But we have shown in the previous paragraph that $M_{1} \subseteq M$, so $u_{1} \in M$. Therefore $u_{2}=u-u_{1} \in M$. Hence $M_{2} \subseteq M$. This gives $M_{1} \oplus M_{2} \subseteq M$ and hence $M=M_{1} \oplus M_{2}$. Thus $M=\left(M \cap E_{1}\right) \oplus\left(M \cap E_{2}\right)$. 
A quasi-injective module is obviously automorphism-invariant. In the next result we give a condition under which an automorphism-invariant module must be quasiinjective.

Theorem 4. Let $M$ be a right module over any ring $R$ such that every summand $E_{1}$ of $E(M)$ admits an automorphism $\sigma_{1}$ such that $I_{E_{1}}-\sigma_{1}$ is also an automorphism of $E_{1}$, then $M$ is automorphism-invariant if and only if $M$ is quasi-injective.

Proof. Let $M$ be automorphism-invariant. Set $E=E(M)$. Suppose every summand $E_{1}$ of $E$ admits an automorphism $\sigma_{1}$ such that $I_{E_{1}}-\sigma_{1}$ is also an automorphism of $E_{1}$.

Let $\sigma \in \operatorname{End}(E)$ be an arbitrary element. Since $\operatorname{End}(E)$ is a clean ring [1, $\sigma=\alpha+\beta$ where $\alpha$ is an idempotent and $\beta$ is an automorphism.

Let $E_{1}=\alpha E$, and $E_{2}=(1-\alpha) E$. Then $E=E_{1} \oplus E_{2}$. By Lemma 3, we have $M=M_{1} \oplus M_{2}$ where $M_{1}=M \cap E_{1}, M_{2}=M \cap E_{2}$.

Then clearly $\alpha(M) \subseteq M$. Since $M$ is automorphism-invariant, $\beta(M) \subseteq M$. Thus $\sigma(M) \subseteq M$. Hence $M$ is quasi-injective.

The converse is obvious.

As a consequence of this theorem, we may now deduce the following which extends the result of Dickson and Fuller [5] to any algebra (not necessarily finitedimensional) over a field $\mathbb{F}$ with more than two elements.

Corollary 5. Let $R$ be any algebra over a field $\mathbb{F}$ with more than two elements. Then the following are equivalent;

(i) Each indecomposable right $R$-module is automorphism-invariant.

(ii) Each indecomposable right $R$-module is quasi-injective, that is, $R$ is of right invariant module type.

Proof. Clearly, for any right $R$-module $E$, the multiplication by an element $u \in \mathbb{F}$ where $u \neq 0$ and $u \neq 1$ gives an automorphism $\sigma$ of $E$ such that $I_{E}-\sigma$ is also an automorphism of $E$. Hence the result follows from the above theorem.

Corollary 6. (14) Let $R$ be a ring in which 2 is invertible. Then any automorphisminvariant module over $R$ is quasi-injective.

Proof. Let $M$ be an automorphism-invariant right $R$-module. Let $E=E(M)$. Let $E_{1}$ be any summand of $E$. We have automorphism $\sigma_{1}: E_{1} \rightarrow E_{1}$, given by $\sigma_{1}(x)=2 x, x \in E_{1}$. Clearly, $I_{E_{1}}-\sigma_{1}=-I_{E_{1}}$ is also an automorphism of $E_{1}$. By Theorem 4, $M$ is quasi-injective.

In the next lemma we give another useful result on decomposition of automorphisminvariant modules.

Lemma 7. Let $M$ be an automorphism-invariant right module over any ring $R$. If $E(M)=E_{1} \oplus E_{2} \oplus E_{3}$, where $E_{1} \cong E_{2}$, then

$$
M=\left(M \cap E_{1}\right) \oplus\left(M \cap E_{2}\right) \oplus\left(M \cap E_{3}\right) .
$$

Proof. Set $E(M)=E$. Let $E=E_{1} \oplus E_{2} \oplus E_{3}$. Let $\sigma: E_{1} \rightarrow E_{2}$ be an isomorphism and let $\pi_{1}: E \rightarrow E_{1}, \pi_{2}: E \rightarrow E_{2}$, and $\pi_{3}: E \rightarrow E_{3}$ be the canonical projections. Then $M \cap E_{1} \subseteq \pi_{1}(M), M \cap E_{2} \subseteq \pi_{2}(M)$ and $M \cap E_{3} \subseteq \pi_{3}(M)$. 
Let $\eta=\sigma^{-1}$. Consider the map $\lambda_{1}: E \rightarrow E$ given by $\lambda_{1}\left(x_{1}, x_{2}, x_{3}\right)=$ $\left(x_{1}, \sigma\left(x_{1}\right)+x_{2}, x_{3}\right)$. Clearly, $\lambda_{1}$ is an automorphism of $E$. Since $M$ is automorphisminvariant, $M$ is invariant under $\lambda_{1}$ and $I_{E}$. Consequently, $M$ is invariant under $\lambda_{1}-I_{E}$. Thus $\left(\lambda_{1}-I_{E}\right)(M) \subseteq M$. Next, we consider the map $\lambda_{2}: E \rightarrow E$ given by $\lambda_{2}\left(x_{1}, x_{2}, x_{3}\right)=\left(x_{1}+\eta\left(x_{2}\right), x_{2}, x_{3}\right)$. This map $\lambda_{2}$ is also an automorphism of $E$. Thus, as explained above, $M$ is invariant under $\lambda_{2}-I_{E}$ too, that is $\left(\lambda_{2}-I_{E}\right)(M) \subseteq M$.

Let $x=\left(x_{1}, x_{2}, x_{3}\right) \in M$. Then $\left(\lambda_{1}-I_{E}\right)(x)=\left(0, \sigma\left(x_{1}\right), 0\right) \in M$. Similarly, we have $\left(\lambda_{2}-I_{E}\right)(x)=\left(\eta\left(x_{2}\right), 0,0\right) \in M$. This gives $\left(\lambda_{1}-I_{E}\right)\left(\eta\left(x_{2}\right), 0,0\right)=$ $\left(0, \sigma \eta\left(x_{2}\right), 0\right)=\left(0, x_{2}, 0\right) \in M$. Thus $\pi_{2}(M) \subseteq M$. Similarly, $\left(\lambda_{2}-I_{E}\right)\left(0, \sigma\left(x_{1}\right), 0\right)=$ $\left(\eta \sigma\left(x_{1}\right), 0,0\right)=\left(x_{1}, 0,0\right) \in M$. Thus $\pi_{1}(M) \subseteq M$. This yields that $\left(0,0, x_{3}\right) \in M$, that is, $\pi_{3}(M) \subseteq M$. This shows that $\pi_{1}(M) \oplus \pi_{2}(M) \oplus \pi_{3}(M) \subseteq M$ and therefore, $M=\pi_{1}(M) \oplus \pi_{2}(M) \oplus \pi_{3}(M)$. Hence $M=\left(M \cap E_{1}\right) \oplus\left(M \cap E_{2}\right) \oplus\left(M \cap E_{3}\right)$.

As a consequence of the above decomposition, we have the following for socle of an indecomposable automorphism-invariant module.

Corollary 8. If $M$ is an indecomposable automorphism-invariant right module over any ring $R$, then $\operatorname{Soc}(M)$ is square-free.

Proof. Let $M$ be an indecomposable automorphism-invariant module. Suppose $M$ has two isomorphic simple submodules $S_{1}$ and $S_{2}$. Then $E(M)=E_{1} \oplus E_{2} \oplus E_{3}$, where $E_{1}=E\left(S_{1}\right), E_{2}=E\left(S_{2}\right)$ and $E_{1} \cong E_{2}$. By Lemma $7 M$ decomposes as $M=\left(M \cap E_{1}\right) \oplus\left(M \cap E_{2}\right) \oplus\left(M \cap E_{3}\right)$, a contradiction to our assumption that $M$ is indecomposable. Hence $\operatorname{Soc}(M)$ is square-free.

Next, we have the following for any indecomposable semi-artinian automorphisminvariant module.

Corollary 9. Let $R$ be any ring and let $M$ be any indecomposable semi-artinian automorphism-invariant right $R$-module. Then one of the following statements holds:

(i) $M$ is uniform and quasi-injective.

(ii) Any simple submodule $S$ of $M$ has identity as its only automorphism.

Proof. Let $M$ be an indecomposable semi-artinian automorphism-invariant right $R$-module. Since $M$ is semi-artinian, $\operatorname{Soc}(M) \neq 0$. By Corollary 8 we know that $\operatorname{Soc}(M)$ is square-free. Suppose $S$ is a simple submodule of $M$. Now $D=\operatorname{End}(S)$ is a division ring.

Suppose $|D|>2$. Then there exists a $\sigma \in D$ such that $\sigma \neq 0$ and $\sigma \neq I_{S}$. Then $I_{S}-\sigma$ is an automorphism of $S$. Let $E=E(M)$ and $E_{1}=E(S) \subseteq E$. Then $E=E_{1} \oplus E_{2}$ for some submodule $E_{2}$ of $E$. Let $\sigma_{1} \in \operatorname{End}\left(E_{1}\right)$ be an extension of $\sigma$. Then $\sigma_{1}$ is an automorphism of $E_{1}$ and $\left(I_{E_{1}}-\sigma_{1}\right)(S)=\left(I_{S}-\sigma\right)(S) \neq 0$. Hence $I_{E_{1}}-\sigma_{1}$ is an automorphism of $E_{1}$. Thus, by Lemma 3 $M=\left(M \cap E_{1}\right) \oplus\left(M \cap E_{2}\right)$. As $M$ is indecomposable, we must have $M=M \cap E_{1}$. Therefore, $M$ is uniform. Then $\operatorname{End}(E(M))$ is a local ring. Therefore for any $\alpha \in \operatorname{End}(E(M)), \alpha$ is an automorphism or $I-\alpha$ is an automorphism. In any case $\alpha(M) \subseteq M$. Therefore $M$ is quasi-injective.

Now, if $M$ is not uniform then $|D|=2$, that is $D=\operatorname{End}(S) \cong \frac{\mathbb{Z}}{2 \mathbb{Z}}$. In this case, the only automorphism of $S$ is the identity automorphism. 
Remark 10. Recall that an algebra $A$ is said to be of finite module type if $A$ has only a finite number of non-isomorphic indecomposable right modules. In regard to Corollary 8, we would like to mention here that Curtis and Jans proved that if A is an algebra over an algebraically closed field $\mathbb{F}$ such that for each indecomposable right $A$-module $M, \operatorname{Soc}(M)$ is square-free, then $A$ is of finite module type (see [4). This was extended by Dickson and Fuller who proved that if $A$ is an algebra over any field $\mathbb{F}$ such that $A$ is of right invariant module type then $A$ has finite module type [5].

We call a ring $R$ to be of right automorphism-invariant type (in short, RAItype), if every finitely generated indecomposable right $R$-module is automorphisminvariant. We would like to understand the structure of right artinian rings of RAI-type.

Lemma 11. Let $R$ be a right artinian ring of RAI-type. Let $e \in R$ be an indecomposable idempotent such that $e R$ is not uniform. Let $A$ be a right ideal contained in $\operatorname{Soc}(e R)$. Then $\operatorname{Soc}(e R)=A \oplus A^{\prime}$ where $A^{\prime}$ has no simple submodule isomorphic to a simple submodule of $A$ and $e R / A^{\prime}$ is quasi-projective.

Proof. As $\operatorname{Soc}(e R)$ is square-free, $\operatorname{Soc}(e R)=A \oplus A^{\prime}$ where $A^{\prime}$ has no simple submodule isomorphic to a simple submodule of $A$. If for some ere $\in e$ Re, ere $A^{\prime} \nsubseteq A^{\prime}$, then for some minimal right ideal $S \subset A^{\prime}$, ere $S \nsubseteq A^{\prime}$. This gives that $S$ is isomorphic to a simple submodule contained in $A$, a contradiction. Hence $e R / A^{\prime}$ is quasi-projective.

Lemma 12. Let $R$ be a right artinian ring of RAI-type. Then any uniserial right $R$-module is quasi-projective.

Proof. Let $A$ be a uniserial right $R$-module with composition length $l(A)=n \geq 2$. We will prove the result by induction. Suppose first that $n=2$. In this case, we can take $J(R)^{2}=0$. For some indecomposable idempotent $e \in R$, we have $A \cong e R / B$ for some $B \subseteq \operatorname{Soc}(e R)$. By Lemma $11, A$ is quasi-projective.

Now consider $n>2$ and assume that the result holds for $n-1$. Let $0 \neq \sigma$ : $A \rightarrow A / C$ be a homomorphism where $C \neq 0$. Suppose $\sigma$ cannot be lifted to a homomorphism $\eta: A \rightarrow A$. Let $F=\operatorname{Soc}(A)$. Then $F \subseteq \operatorname{Ker}(\sigma)$. We get a mapping $\bar{\sigma}: \frac{A}{F} \rightarrow \frac{A}{C}$. By the induction hypothesis, there exists a homomorphism $\bar{\eta}: \frac{A}{F} \rightarrow \frac{A}{F}$ such that $\bar{\sigma}=\pi \bar{\eta}$, where $\pi: \frac{A}{F} \rightarrow \frac{A}{C}$ is a natural homomorphism.

Let $M=A \times A$, and $N=\{(a, b) \in M: \bar{\eta}(a+F)=b+F\}$. Then $N$ is a submodule of $M$. Now there exist elements $x \in A$ and indecomposable idempotent $e \in R$ such that $A=x R$ and $x e=x$. Fix an element $y \in A$ such that $\bar{\eta}(x+F)=y+F$ and $y e=y$. Set $z=(x, y)$. Then $z \in N$ and $N_{1}=z R$ is local. Let $\pi_{1}, \pi_{2}$ be the associated projections of $M$ onto the first and second components of $M$, respectively. Then $\pi_{1}\left(N_{1}\right)=A$.

Now, we claim that $N_{1}$ is uniserial. If $N_{1}$ is not uniform, then $\operatorname{Soc}\left(N_{1}\right)=\operatorname{Soc}(M)$. Therefore $\operatorname{Soc}\left(N_{1}\right)$ is not square-free, which is a contradiction by Lemma 8 . Thus $N_{1}$ is uniform. It follows that $N_{1}$ embeds in $A$ under $\pi_{1}$ or $\pi_{2}$. Hence $N_{1}$ is uniserial. As $\pi_{1}\left(N_{1}\right)=A$, and $l\left(N_{1}\right) \leq l(A)$, it follows that $\left.\pi_{1}\right|_{N_{1}}$ is an isomorphism. Thus given any $x \in A$, there exists a unique $y \in A$ such that $(x, y) \in N_{1}$. We get a homomorphism $\lambda: A \rightarrow A$ such that $\lambda(x)=y$ if and only if $(x, y) \in N_{1}$. Clearly $\lambda$ lifts $\bar{\eta}$ and hence it also lifts $\sigma$. This proves that $A$ is quasi-projective. 
Lemma 13. Let $R$ be a right artinian ring of RAI-type. Let $A_{R}$ be any uniserial module. Then the rings of endomorphisms of different composition factors of $A$ are isomorphic.

Proof. Let $A$ be a uniserial right $R$-module with $l(A)=2$. Let $C=a n n_{r}(A)$ and $\bar{R}=R / C$. As $A_{R}$ is quasi-projective, $A$ is a projective $\bar{R}$-module. Thus there exists an indecomposable idempotent $e \in R$ such that $A \cong \bar{e} \bar{R}$. As $\bar{R}$ embeds in a finite direct sum of copies of $A$, there exists an indecomposable idempotent $f \in R$ such that $\operatorname{Soc}(A) \cong \frac{\overline{f R}}{\overline{f J(R)}}, \bar{e} \overline{J(R)}=\overline{e x f R}$ for some $x \in J(R)$. We get an embedding $\sigma: \frac{e R e}{e J(R) e} \rightarrow \frac{f R f}{f J(R) f}$ defined as $\sigma($ ere $+e J(R) e)=f r^{\prime} f+f J(R) f$ whenever $\overline{\text { ereexf }}=\overline{e x f f r^{\prime} f}$; ere $\in e R e, f r^{\prime} f \in f R f$. Let $z=f v f \in f R f$. We get an $\bar{R}$-homomorphism $\eta: \bar{e} \overline{J(R)} \rightarrow \bar{e} \overline{J(R)}$ such that $\eta(\overline{e x f})=\overline{e x f f v f}$. As $\bar{e} \bar{R}$ is quasi-injective, there exists an $\bar{R}$-homomorphism $\lambda: \bar{e} \bar{R} \rightarrow \bar{e} \bar{R}$ extending $\eta$. Now $\lambda(\bar{e})=\overline{e r e}$ for some $r \in R$. Then $\overline{\text { ere } \overline{e x f}}=\lambda(\overline{e x f})=\eta(\overline{e x f})=\overline{e x f f v f}$, which gives that $\sigma$ is onto. Hence $\frac{e R e}{e J(R) e} \cong \frac{f R f}{f J(R) f}$. Thus the result holds whenever $l(A)=2$. If $l(A)=n>2$, the result follows by induction on $n$.

Lemma 14. Let $R$ be a right artinian ring of RAI-type. Then we have the following.

(i) Let $D$ be a division ring and $x \in R$. Let $x R$ be a local module such that for any simple submodule $S$ of $\operatorname{Soc}(x R), D=\operatorname{End}(S)$. Then $\operatorname{End}(x R / x J(R)) \cong D$.

(ii) Let $x R$ be a local module and $D=\operatorname{End}(x R / x J(R))$ where $x \in R$. Then $\operatorname{End}(S) \cong D$ for every composition factor $S$ of $x R$.

(iii) Let $x R, y R$ be two local modules where $x, y \in R$. If $\operatorname{End}(x R / x J(R)) \nsucceq$ $\operatorname{End}(y R / y J(R))$, then $\operatorname{Hom}(x R, y R)=0$.

Proof. (i) There exists an $n \geq 1$ such that $x J(R)^{n}=0$ but $x J(R)^{n-1} \neq 0$. If $n=$ 1 , then $x R$ is simple, so the result holds. We apply induction on $n$. Suppose $n>1$ and assume that the result holds for $n-1$. Now $x J(R) J(R)^{n-1}=0$, but $x J(R) J(R)^{n-2} \neq 0$. Therefore there exists an element $y \in x J(R)$ such that $y R$ is local and $y J(R)^{n-1}=0$ but $y J(R)^{n-2} \neq 0$. By the induction hypothesis, $\operatorname{End}(y R / y J(R)) \cong D$. In fact, for any simple submodule $S^{\prime}$ of $e J(R) / x J(R)^{2}, \operatorname{End}\left(S^{\prime}\right) \cong D$. Consider the local module $M=x R / x J(R)^{2}$. Let $S^{\prime}$ be a simple submodule of $M$. Then $\operatorname{Soc}(M)=S^{\prime} \oplus B$ for some $B \subset \operatorname{Soc}(M)$. Then $\operatorname{End}\left(S^{\prime}\right) \cong D$. As $A=M / B$ is uniserial, $\operatorname{Soc}(A) \cong S^{\prime}$ and $A / A J(R) \cong x R / x J(R)$. By Lemma 13 End $(x R / x J(R)) \cong D$.

(ii) Let $S$ be a simple submodule of $\operatorname{Soc}(x R)$ and $B$ be a complement of $S$ in $x R$. Then $\overline{x R}=x R / B$ is uniform and $\operatorname{Soc}(\overline{x R}) \cong S$. By (i), $\operatorname{End}(S) \cong$ $\operatorname{End}\left(\frac{\overline{x R}}{\overline{x J(R)}}\right) \cong \operatorname{End}(x R / x J(R))=D$. Hence $\operatorname{End}(S) \cong D$ for any simple submodule $S$ of $x R$. Let $S_{1}$ be any composition factor of $x R$. Then there exists a local submodule $y R$ of $x R$ such that $S_{1} \cong y R / y J(R)$. By (i), $\operatorname{End}\left(S_{1}\right) \cong$ $\operatorname{End}(S) \cong D$, where $S$ is a simple submodule of $y R$.

(iii) It is immediate from (ii). 
Now, we are ready to give the structure of indecomposable right artinian rings of RAI-type.

Theorem 15. Let $R$ be an indecomposable right artinian ring of RAI-type. Then the following hold.

(i) There exists a division ring $D$ such that $\operatorname{End}(S) \cong D$ for any simple right $R$-module $S$. In particular, $R / J(R)$ is a direct sum of matrix rings over $D$.

(ii) If $D \neq \mathbb{Z} / 2 \mathbb{Z}$, then every finitely generated indecomposable right $R$-module is quasi-injective. In this case, $R$ is right serial.

Proof. (i) Let $e \in R$ be an indecomposable idempotent and $D=e R e / e J(R) e$. By above lemma, every composition factor $S$ of $e R$ satisfies $\operatorname{End}(S) \cong D$. Now $R_{R}=\oplus_{i=1}^{n} e_{i} R$ where $e_{i}$ are orthogonal indecomposable idempotents with $e_{1}=e$. Let $A$ be the direct sum of those $e_{j} R$ for which $\frac{e_{j} R e_{j}}{e_{j} J(R) e_{j}} \cong D$. Consider any $e_{k}$ for which $\frac{e_{k} R e_{k}}{e_{k} J(R) e_{k}} \neq D$. It follows from Lemma 14(iii) that $A e_{k} R=0=e_{k} R A$. Consequently, $A=e_{k} R$ and we get that $R=A \oplus B$ for some ideal $B$. As $R$ is indecomposable, we get $R=A$. This proves (i).

(ii) Suppose $D \neq \mathbb{Z} / 2 \mathbb{Z}$. It follows from Corollary 9 that every indecomposable right $R$-module is uniform and quasi-injective. In particular, if $e \in R$ is an indecomposable idempotent, then any homomorphic image of $e R$ is uniform, which gives that $e R$ is uniserial. Hence $R$ is right serial.

Theorem 16. 16 Let $R$ be a right artinian ring such that $J(R)^{2}=0$. If every finitely generated indecomposable right $R$-module is local, then $R$ satisfies the following conditions.

(a) Every uniform right $R$-module is either simple or is injective with composition length 2.

(b) $R$ is a left serial ring.

(c) For any indecomposable idempotent $e \in R$ either eJ $(R)$ is homogeneous or $l(e J(R)) \leq 2$.

Conversely, if $R$ satisfies (a), (b), (c) and $l(e J(R)) \leq 2$, then every finitely generated indecomposable right $R$-module is local.

Example. Let $R=\left[\begin{array}{ccc}\mathbb{F} & \mathbb{F} & \mathbb{F} \\ 0 & \mathbb{F} & 0 \\ 0 & 0 & \mathbb{F}\end{array}\right]$ where $\mathbb{F}=\frac{\mathbb{Z}}{2 \mathbb{Z}}$.

Then $R$ is a left serial ring. We have already seen that $e_{11} R$ is an indecomposable module which is automorphism-invariant but not quasi-injective. It follows from Theorem 16 that every finitely generated indecomposable right $R$-module is local. Thus the only indecomposable modules which are not simple are the homomorphic images of $e_{11} R$, which are $e_{11} R, \frac{e_{11} R}{e_{12} \mathbb{F}}$, and $\frac{e_{11} R}{e_{13} \mathbb{F}}$. These are all automorphisminvariant. It follows from Theorem [16 that any finitely generated indecomposable right $R$-module is local. Thus this ring $R$ is an example of a ring where every finitely generated indecomposable right $R$-module is automorphism-invariant. 
Example. Let $\mathbb{F}=\mathbb{Z} / 2 \mathbb{Z}$ and $R=\left[\begin{array}{cccc}\mathbb{F} & \mathbb{F} & \mathbb{F} & \mathbb{F} \\ 0 & \mathbb{F} & 0 & 0 \\ 0 & 0 & \mathbb{F} & 0 \\ 0 & 0 & 0 & \mathbb{F}\end{array}\right]$.

This ring $R$ is left serial and $J(R)^{2}=0$. Now $e_{11} J(R)=e_{12} \mathbb{F} \oplus e_{13} \mathbb{F} \oplus e_{14} \mathbb{F}$, a direct sum of non-isomorphic minimal right ideals. It follows from condition (c) in Theorem 16 that there exists a finitely generated indecomposable right $R$-module that is not local. We have $E_{1}=E\left(e_{12} \mathbb{F}\right), E_{2}=E\left(e_{13} \mathbb{F}\right), E_{3}=E\left(e_{14} \mathbb{F}\right)$, each of them has composition length 2. Now $e_{11} R$ has two homomorphic images $A_{1}=\frac{e_{11} R}{e_{14} \mathbb{F}}$ and $A_{2}=\frac{e_{11} R}{e_{12} \mathbb{F}}$ such that $\operatorname{Soc}\left(A_{1}\right) \cong e_{12} \mathbb{F} \oplus e_{13} \mathbb{F}$ and $\operatorname{Soc}\left(A_{2}\right) \cong e_{13} \mathbb{F} \oplus e_{14} \mathbb{F}$. So we get $B_{1} \subseteq E_{1} \oplus E_{2} \subseteq E_{1} \oplus E_{2} \oplus E_{3}$ such that $A_{1} \cong B_{1}$. Similarly, we have $A_{2} \cong B 2 \subseteq E_{2} \oplus E 3$. Let $E=E_{1} \oplus E 2 \oplus E_{3}$. Its only automorphism is $I_{E}$. Thus any essential submodule of $E$ is automorphism-invariant. Now $B=$ $B_{1}+B_{2} C_{e} E$, so $B$ is automorphism-invariant and $B$ is not local. We prove that $B$ is indecomposable. We have $B_{1} \cap B_{2}=e_{13} \mathbb{F}$. Notice that any submodule of $E_{1} \oplus E_{2}$ that is indecomposable and not uniserial is $B_{1}$. Suppose a simple submodule $S$ of $B$ is a summand of $B$. But $S \subset B_{1}$ or $S \subset B_{2}$, therefore $B_{1}$ or $B_{2}$ decomposes, which is a contradiction. As $l(B)=5, B$ has a summand $C_{1}$ with $l\left(C_{1}\right)=2$. Then $C_{1}$ being uniserial, it equals one of $E_{i}$.

Case 1. $C_{1}=E_{1}$. Then $B=C_{1} \oplus C_{2}$, where $\operatorname{Soc}\left(C_{2}\right) \cong B_{2}$. As $C_{2}$ has no uniserial submodule of length 2 , the projection of $B_{1}$ in $C_{2}$ equals $\operatorname{Soc}\left(C_{2}\right)$, we get $B_{1}$ is semi-simple, which is a contradiction.

Similarly other cases follow. Hence $B$ is indecomposable.

Now, we proceed to answer the question of Lee and Zhou [14] whether every automorphism-invariant module is pseudo-injective in the affirmative for modules with finite Goldie dimension.

Theorem 17. If $M$ is an automorphism-invariant module with finite Goldie dimension, then $M$ is pseudo-injective.

Proof. Let $N$ be a submodule of $M$. Let $\sigma: N \rightarrow M$ be a monomorphism. Then $\sigma$ can be extended to a monomorphism $\sigma^{\prime}: E(N) \rightarrow E(M)$. Now, we may write $E(M)=\sigma^{\prime}(E(N)) \oplus P=E(N) \oplus Q$ for some submodules $P$ and $Q$ of $E(M)$. Note that $\sigma^{\prime}(E(N)) \cong E(N)$. Since $M$ has finite Goldie dimension, $E(M)$ has finite Goldie dimension. Thus $E(M)$ is a directly-finite injective module, and hence $E(M)$ satisfies internal cancellation property. Therefore, $P \cong Q$. Thus, there exists an isomorphism $\varphi: Q \rightarrow P$. Now consider the mapping $\lambda: E(M) \rightarrow E(M)$ defined as $\lambda(x+y)=\sigma^{\prime}(x)+\varphi(y)$ where $x \in E(N)$ and $y \in Q$. Clearly, $\lambda$ is an automorphism of $E(M)$. Since $M$ is assumed to be automorphism-invariant, we have $\lambda(M) \subseteq M$. Thus $\left.\lambda\right|_{M}: M \rightarrow M$ extends $\sigma$. This shows that $M$ is pseudo-injective.

It is well known that if $R$ is a ring such that each cyclic right $R$-module is injective then $R$ is semisimple artinian. For more details on rings characterized by properties of their cyclic modules, the reader is referred to [9]. We would like to understand the structure of rings for which each cyclic module is automorphism-invariant. In [14] it is shown that if every 2-generated right module over a ring $R$ is automorphisminvariant, then $R$ is semisimple artinian. 
A ring $R$ is called a right SI ring if every singular right $R$-module is injective [ 6 . In [8] Huynh, Jain, and López-Permouth proved that a simple ring $R$ is a right SI ring if and only if every cyclic singular right $R$-module is $\pi$-injective. Their proof can be adapted to show that a simple right noetherian ring $R$ is a right SI ring if and only if every cyclic singular right $R$-module is automorphism-invariant.

The following lemma due to Huynh et al [7, Lemma 3.1] is crucial for proving our result. This lemma is, in fact, a generalization of a result of J. T. Stafford given in [2, Theorem 14.1].

Lemma 18. ([7]) Let $R$ be a simple right Goldie ring which is not artinian and $M$ a torsion right $R$-module. If $M=a R+b R$, where $b R$ is of finite composition length and $f$ is a non-zero element of $R$ then $M=(a+b x f) R$ for some $x \in R$.

We know that for a prime right Goldie ring $R$, singular right $R$-modules are the same as torsion right $R$-modules. Now, we are ready to prove the following.

Theorem 19. Let $R$ be a simple right noetherian ring. Then $R$ is a right SI ring if and only if every cyclic singular right $R$-module is automorphism-invariant.

Proof. Let $R$ be a simple right noetherian ring such that every cyclic singular right $R$-module is automorphism-invariant. We wish to show that $R$ is a right SI ring. If $\operatorname{Soc}\left(R_{R}\right) \neq 0$, then as $R$ is a simple ring, $R=\operatorname{Soc}\left(R_{R}\right)$ and hence $R$ is simple artinian.

Now, assume $\operatorname{Soc}\left(R_{R}\right)=0$. Let $M$ be any artinian right $R$-module. Since any module is homomorphic image of a free module, we may write $M \cong F / K$ where $F$ is a free right $R$-module. We first claim that $K$ is an essential submodule of $F$. Assume to the contrary that $K$ is not essential in $F$. Let $T$ be a complement of $K$ in $F$. Note that $M \cong \frac{F}{K} \supset \frac{K \oplus T}{K} \cong T$. Since $M$ is an artinian module, $\operatorname{Soc}(M) \neq 0$ and consequently $\operatorname{Soc}(T) \neq 0$. This yields that $\operatorname{Soc}(F) \neq 0$, a contradiction to the assumption that $\operatorname{Soc}\left(R_{R}\right)=0$. Therefore, $K$ is an essential submodule of $F$ and hence $M$ is a singular module. Let $C$ be a cyclic submodule of $M$. We have $\operatorname{Soc}(C) \neq 0$. As $R$ is right noetherian and $C$ is a cyclic right $R$-module, $C$ is noetherian. Thus we have $\operatorname{Soc}(C)=\oplus_{i=1}^{k} S_{i}$ where each $S_{i}$ is a simple right $R$ module. By the above lemma, it follows that $C \oplus S_{1}$ is cyclic. By induction, it may be shown that $C \oplus \operatorname{Soc}(C)$ is cyclic. Now as $C \oplus \operatorname{Soc}(C)$ is a cyclic singular right $R$-module, by assumption $C \oplus \operatorname{Soc}(C)$ is automorphism-invariant. Hence $\operatorname{Soc}(C)$ is $C$-injective. Therefore, $\operatorname{Soc}(C)$ splits in $C$ and hence $C=\operatorname{Soc}(C) \subset M$. Thus $M$ is semisimple. This shows that any artinian right $R$-module $M$ is semisimple.

Now, we prove that every singular module over $R$ is semisimple, or equivalently, for each essential right ideal $E$ of $R, R / E$ is semisimple. By the above claim, it suffices to show that $R / E$ is artinian. Set $N=R / E$. If $N$ is not artinian, then we get $0 \subset V_{1} \subset N$ with $V_{1}$ not artinian. Now $N$ is torsion, so is $V_{1}$. Therefore, $Q=$ $N \oplus V_{1}$ is torsion and hence cyclic by Lemma 18. Thus we can write $x R=N \oplus V_{1}$ for some $x \in R$. By the assumption, $x R$ is automorphism-invariant. Hence $V_{1}$ is $N$-injective. So $N=N_{1} \oplus V_{1}$. Repeat the process with $V_{1}$, so $V_{1}=N_{2} \oplus V_{2}$, where $N_{2} \neq 0$ and $V_{2}$ is not artinian. Continuing this process, we get an infinite direct sum of $N_{i}$ in $N$, which is a contradiction. Thus we conclude that any singular right $R$-module is artinian and consequently semisimple. 
Thus $R$ is a right nonsingular ring such that every singular right $R$-module is semisimple. Hence, by [6], $R$ is a right SI ring.

The converse is obvious.

\section{Questions}

Question 1: Does every automorphism-invariant module satisfy the property C2 ?

Lee and Zhou [14 have shown that every automorphism-invariant module satisfies the property $\mathrm{C} 3$.

Question 2: What is example of an automorphism-invariant module which is not pseudo-injective?

In Theorem 17 above, we have shown that such a module cannot have finite Goldie dimension.

A module $M$ is called skew-injective if for every submodule $N$ of $M$, any endomorphism of $N$ extends to an endomorphism of $M$. In [9] it is asked whether every skew-injective module with essential socle is quasi-injective. We ask the following

Question 3: Is every automorphism-invariant module with essential socle a quasiinjective module?

Call a ring $R$ to be a right a-ring if each right ideal of $R$ is automorphism-invariant.

Question 4: Describe the structure of a right a-ring.

Call a ring $R$ to be a right $\Sigma$-a-ring if each right ideal of $R$ is a finite direct sum of automorphism-invariant right ideals.

Question 5: Describe the structure of a right $\Sigma$-a-ring.

Question 6: Let $R$ be a simple ring such that $R_{R}$ is automorphism-invariant. Must $R$ be a right self-injective ring?

In fact, this question is open even when $R_{R}$ is pseudo-injective (see [3]). 


\section{REFERENCES}

[1] V. P. Camillo, D. Khurana, T. Y. Lam, W. K. Nicholson, Y. Zhou, Continuous modules are clean, J. Algebra, 304 (2006), 94-111.

[2] A. W. Chatters, C. R. Hajarnavis, Rings with Chain Conditions, Pitman, London, 1980.

[3] J. Clark and D.V. Huynh, Simple rings with injectivity conditions on one-sided ideals, Bull. Australian Math. Soc. 76 (2007), 315-320.

[4] C. W. Curtis and J. P. Jans, On algebras with a finite number of indecomposable modules, Trans. Amer. Math. Soc. 114 (1965), 122-132.

[5] S. E. Dickson, K. R. Fuller, Algebras for which every indecomposable right module is invariant in its injective envelope, Pacific J. Math., vol. 31, no. 3 (1969), 655-658.

[6] K. R. Goodearl, Singular torsion and the splitting properties, Memoirs Amer. Math. Soc. $124,1972$.

[7] D. V. Huynh, S. K. Jain, S. R. López-Permouth, When is a simple ring noetherian?, J. Algebra, 184 (1996), 784-794.

[8] D. V. Huynh, S. K. Jain, S. R. López-Permouth, When cyclic singular modules over a simple ring are injective, J. Algebra, 263 (2003), 185-192.

[9] S. K. Jain, A. K. Srivastava and A. A. Tuganbaev, Cyclic Modules and the Structure of Rings, Oxford Mathematical Monographs, Oxford University Press, 2012.

[10] J. P. Jans, On the indecomposable representations of algebras, Ann. of Math. (2) 66 (1957), 418-429.

[11] T. Y. Lam, A First Course in Noncommutative Rings, Second Edition, Springer-Verlag, 2001.

[12] T. Y. Lam, Lectures on Modules and Rings, Springer-Verlag, 1999.

[13] T. Y. Lam, A crash course on stable range, cancellation, substitution and exchange, J. Alg. Appl., vol. 3, no. 3 (2004), 301-343.

[14] T. K. Lee, Y. Zhou, Modules which are invariant under automorphisms of their injective hulls, J. Alg. Appl., to appear.

[15] S. H. Mohamed, B. J. Müller, Continuous and Discrete Modules, London Math. Soc. Lecture Note Ser., vol. 147, Cambridge Univ. Press, Cambridge, 1990.

[16] S. Singh, H. Al- Bleehed, Rings with indecomposable modules local, Beiträge zur Algebra und Geometrie, 45 (2005), 239-251.

[17] S. Singh, A. K. Srivastava, Dual automorphism-invariant modules, preprint.

[18] M. L. Teply, Pseudo-injective modules which are not quasi-injective, Proc. Amer. Math. Soc., vol. 49, no. 2 (1975), 305-310.

House No. 424, Sector No. 35 A, Chandigarh-160036, India

E-mail address: ossinghpal@yahoo.co.in

Department of Mathematics and Computer Science, St. Louis University, St. Louis, MO-63103, USA

E-mail address: asrivas3@slu.edu 